\title{
Integrating Patient-Specific Electrocardiogram Signals and Image-Based Computational Fluid Dynamics Method to Analyze Coronary Blood Flow in Patients during Cardiac Arrhythmias
}

\author{
Szu-Hsien Chou ${ }^{1,2} \cdot$ Kuan-Yu Lin $^{1} \cdot$ Zhen-Ye Chen ${ }^{3} \cdot$ Chun-Jung Juan ${ }^{2} \cdot$ Chien-Yi Ho ${ }^{4} \cdot$ Tzu-Ching Shih $^{1,3}$ (1)
}

Received: 12 July 2019 / Accepted: 10 December 2019 / Published online: 19 December 2019

(c) The Author(s) 2019

\begin{abstract}
Purpose The aim of this study was to use the computational fluid dynamics (CFD) method, patient-specific electrocardiogram (ECG) signals, and computed tomography three-dimensional image reconstruction technique to investigate the blood flow in coronary arteries during cardiac arrhythmia.

Methods Two patients with premature ventricular contraction-type cardiac arrhythmia and one with atrial fibrillation-type cardiac arrhythmia were investigated. The inlet velocity of the coronary artery in simulation was applied with the measured velocity profile of the left ventricular outflow tract (LVOT) from the Doppler echocardiography. The measured patient central aortic blood pressure waveform was employed for the coronary artery outlet in simulation. The no-slip boundary condition was applied to the arterial wall.

Results For the patient with irregular cardiac rhythms (Case I), the coronary blood flow rate under the shortened and lengthened cardiac rhythms were 0.66 and $0.96 \mathrm{~mL} / \mathrm{s}$, respectively. In Case II, the maximum velocity at the LVOT under a normal heartbeat was found to be $101 \mathrm{~cm} / \mathrm{s}$, whereas the average value was $73 \mathrm{~cm} / \mathrm{s}$. In Case III, the patient was also diagnosed with a congenital stenosis problem at the myocardial bridge (MCB) at the LAD. The measured blood flow rate at the MCB of the LAD for the three heartbeats in Case III was found to be $0.68,1.08$, and $1.14 \mathrm{~mL} / \mathrm{s}$.

Conclusion The integration of patient-specific ECG signals and image-based CFD methods can clearly analyze hemodynamic information for patients during cardiac arrhythmia. The cardiac arrhythmia can reduce the blood flow in the coronary arteries.
\end{abstract}

Keywords Electrocardiogram (ECG) · Computational fluid dynamics (CFD) · Coronary blood flow · Arrhythmias · Computed tomographic images

Szu-Hsien Chou and Chun-Jung Juan contributed to this work equally.

\section{Tzu-Ching Shih}

shih@mail.cmu.edu.tw

1 Department of Biomedical Imaging and Radiological Science, China Medical University, 91 Hsueh-Shih Road, Taichung 40402, Taiwan

2 Department of Medical Imaging, China Medical University Hsinchu Hospital, China Medical University, Hsinchu, Taiwan

3 The Ph.D. Program for Medical Engineering and Rehabilitation Science, China Medical University, Taichung, Taiwan

4 Department of Family Medicine, China Medical University Hsinchu Hospital, Hsinchu, Taiwan

\section{Introduction}

Cardiovascular disease (CVD) is a major cause of morbidity and mortality worldwide, with the lifetime risk exceeding $60 \%$ [1]. Globally, the number of deaths due to CVD has also increased by $12.5 \%$ in the past decade [2]. Coronary heart disease is the leading cause of deaths attributable to CVD in the United States (43.8\%) [1]. In particular, coronary artery disease (CAD) is ranked second among the cardiac diseases. Generally, the most common clinical methods for CAD diagnosis are noninvasive coronary computed tomography (CT) angiography (CCTA) and invasive coronary angiography (ICA) [3]. Currently, ICA is considered the gold standard for coronary artery examination. However, ICA can only provide an anatomical evaluation of cardiovascular stenosis. It cannot clearly indicate the relationship between a coronary artery and myocardial ischemia. 
Therefore, Pijls et al. developed an indicator for the functional assessment of the coronary artery hemodynamics, called fractional flow reserve (FFR) [4-9]. However, none of the aforementioned methods, including CCTA, ICA, and FFR, can evaluate the blood flow under cardiac arrhythmia conditions. At this stage, clinical diagnosis and treatment of problems, such as cardiac arrhythmia, can focus only on the transmission direction of the cardiac electrical signals. The effects of blood flow at the coronary arteries during cardiac arrhythmia require further understanding.

Cardiac arrhythmia, characterized by heart rhythm disruption, is caused by abnormal electrical signals in the heart. Premature ventricular contractions (PVCs), which are the most common form of cardiac arrhythmias, can be detected using the electrocardiogram (ECG) signal analysis. PVC is a common event occurring in a person of any age but more frequent in elderly people where the heart beat is initiated by the heart ventricles that are independent of the pace set by the sinoatrial node. Furthermore, atrial fibrillation (AF) is the most prevalent cardiac arrhythmia, the occurrence of which increases with age. The electrocardiogram (ECG), which measures the electric activity of the heart from the body surface and reveals the heart functions, is widely used a tool by cardiologists for determining the abnormalities of the human heart [10-15]. These heart activities in the generation of P-QRS-T waves featuring specific characteristics in terms of amplitude and duration. Irregular cardiac rhythm, also known as cardiac arrhythmia, refers to the different symptoms caused by the abnormalities of the cardiac electrical signal transmission. Accurate detection and classification of life-threatening ventricular arrhythmia episodes such as ventricular fibrillation and rapid ventricular tachycardia from ECG signals is a challenging issue. However, because ECG signals are unsteady and existing signal processing methods lead to relatively high computational burden, automated and accuracy diagnoses remain challenging.

Computation fluid dynamics (CFD) is a technique that uses numerical methods to solve the governing equations of fluid dynamics in a computer and predict the flow field [16-19]. The technique has been applied in the aerospace and automobile industry for more than 50 years. However, it has only been applied to the medical field in the last 10 years to evaluate the blood flow within the human circulatory system [16, 17]. CFD has also been applied in simulating the blood flow in coronary arteries [18]. Miyoshi et al. [19] used CFD techniques to compute the fractional flow reserve from CCTA images $\left(\mathrm{FFR}_{\mathrm{CT}}\right)$, a noninvasive examination. The results indicated that $\mathrm{FFR}_{\mathrm{CT}}$ has a considerable degree of accuracy [19-23]. However, how the different phases of patient cardiac arrhythmias influence coronary blood flow is not yet completely understood. Furthermore, Lin et al. [24] demonstrated that the CFD can be used to simulate coronary blood flow based on patient-specific CT images with different theoretical models of blood flow. Therefore, the aim of this work is to investigate the hemodynamics of coronary blood flow for patients during cardiac arrhythmia using the patient-specific ECG signals and the image-based CFD method.

\section{Materials and Methods}

Three cardiac arrhythmia patients were examined. Two of them were diagnosed with premature ventricular contractions (PVCs) (i.e. PVC-type) cardiac arrhythmia and one was diagnosed with atrial fibrillation (AF) (i.e. AF-type) cardiac arrhythmia. All patient ECG signals were simultaneously recorded during CT scan acquisition. Meanwhile, recorded ECG signals of three cardiac arrhythmia patients were contained with three to four consecutive heartbeats for analysis.

\subsection{CT Imaging and Geometry Reconstruction}

The CT images of the patients were obtained from a 640-sliced (320-detector) CT scanner (Aquilion ONE, Toshiba Medical Systems Corporation). The CT scanner used a slice thickness of $0.5 \mathrm{~mm}$, interval of $0.25 \mathrm{~mm}$, rotation speed of 0.35 s/rotation, and a matrix size of $512 \times 512$. Three-dimensional reconstructions of the obtained coronary artery images were created using the Toshiba Vitrea workstation. The processed 3D geometrical models were output into the STL (i.e. StereoLithography) file format. The coronary artery surface geometries were imported into the ANSYS ICEM-CFD.

According to Kwon et al. [25], the computation time for the coronary artery flow simulation can be greatly reduced when the computation only includes the left and right coronary arteries but excludes the aorta. The difference in the simulated FFR and those of the model including the aorta is $<2 \%$. Therefore, the simplified model in this study also excluded the aorta for better computation time efficiency.

\subsection{Volume Meshing}

Volume meshes of coronary arteries were generated with the meshing module in the ANSYS. The volume mesh was created by applying the unstructured tetrahedral mesh in this study. We performed the grid independence test using the laminar flow model and the pressure gradient difference of the right coronary artery for Case I was displayed in Table 1 . Considering the balance between the numerical accuracy and the computational time, the coronary artery flow model in this study has more than 2.5 million computation grids for each case. 
Table 1 Grid independence test

\begin{tabular}{llllll}
\hline$\Delta \mathrm{P}(\mathrm{mmHg})$ at different flow models/elements $\left(\times 10^{5}\right)$ & 3.5 & 7.4 & 25.2 & 54.8 & 65.9 \\
$\Delta \mathrm{P}(\mathrm{mmHg})$ at laminar & 20.25 & 22.37 & 25.69 & 26.01 & 26.14 \\
\hline
\end{tabular}

Pressure gradient difference of the right coronary artery in Case I

\subsection{Boundary Conditions and Parameter Settings}

In this study, the inlet velocity transient profile of the coronary artery in simulation was used with the measured velocity profile of each patient LVOT by the Doppler echocardiography. However, because the simulation of this study excluded the aorta, it is necessary to redistribute $4 \%$ of the cardiac output ( $\mathrm{CO}$, estimated from the output from the left ventricle to the aorta) to the coronary arteries according to the body circulation $[18,26]$. The redistributed flow of the left and right coronary arteries was then directly set at the entry point of the corresponding arteries [22]. The inlet velocity waveforms were determined according to the transformed periodic wave patterns from the electrocardiographic waveform obtained during the patient's CCTA examination (Fig. 1). The periodic wave patterns were transformed into a function using MATLAB (Fig. 1) and were set at the entrance of the coronary arteries on both sides.

In order to guarantee that the boundary conditions do not affect the blood flow in the region of interest, a cylindrical extension was added to the vessel inflow and outflow (Fig. 1c). The length $\left(\mathrm{L}_{\mathrm{e}}\right)$ added to the vessel inflow and outflow was estimated using a semi-empirical law valid for laminar flow [27]:

$\frac{\mathrm{L}_{\mathrm{e}}}{\mathrm{D}} \approx 0.06 \mathrm{Re}$,

where $\mathrm{D}$ is the diameter of the vessel and Re is the dimensionless Reynolds number.

The transient waveforms of the aortic pressure of the patients were imposed at as the outlet boundary condition. (a)

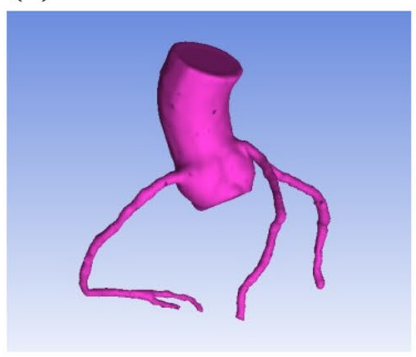

(b)

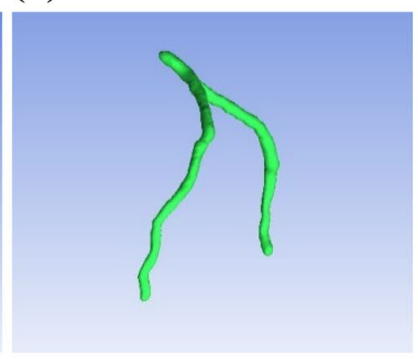

(e)

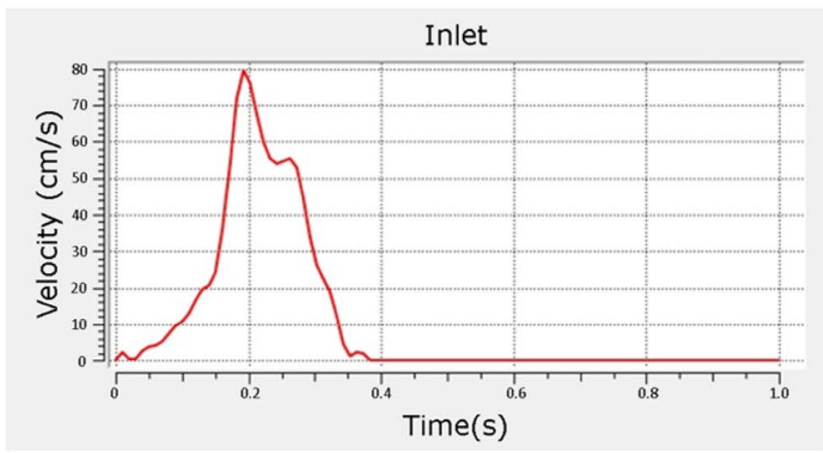

Fig. 1 a Patient-specific coronary surface geometry of the acquired STL from the Toshiba Vitrea workstation; $\mathbf{b}$ coronary arteries without the aorta; $\mathbf{c}$ adding the inlet and outlets extensions. The inlet entrance length of the LCA was $540 \mathrm{~mm}$. The outlet flow extension lengths of D1, and LCX were approximately 82.5 and $50 \mathrm{~mm}$, respectively. d (c)

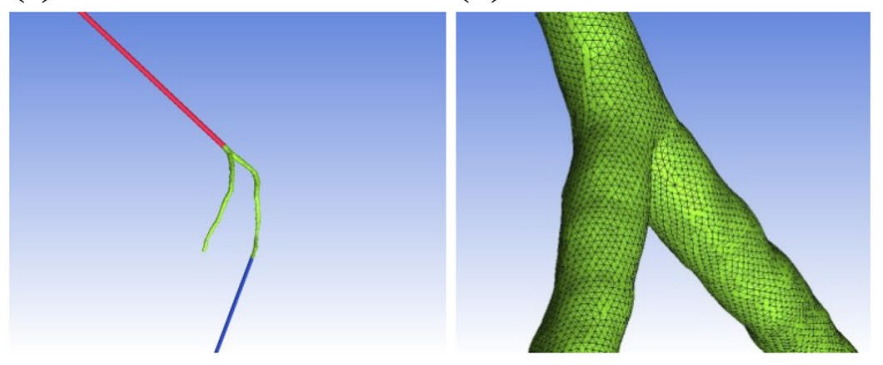

(f)

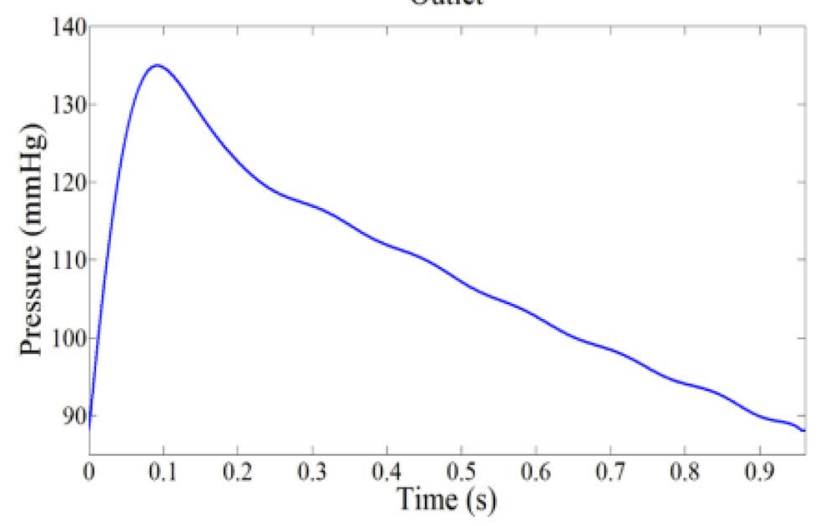

Meshing of the coronary arteries; e velocity profile of the coronary artery for the inlet boundary condition, whereas velocity profile in the LVOT from the Doppler echocardiography measurement. f Pressure profile of the coronary arteries for the outlet boundary condition 
In general, the flow in a circular pipe is laminar for $\operatorname{Re} \leq 2,300$, turbulent for $\operatorname{Re} \geq 4000$, and transitional in between. When the Reynolds Number is less than 2300, the effect of the viscous force to the blood flow is greater than that of inertia. The Reynolds numbers in this study cases are less than 1000 that the laminar flow is appropriate. In other words, the Reynolds number is small enough that laminar flow description is valid. In this case, at small or moderate Reynolds numbers, the viscous forces are large enough to suppress these fluctuations and to keep the fluid in line. The blood flow is laminar when the Reynolds number is small. Thus, the laminar flow model was used. Furthermore, the FFR $_{\text {CTS }}$ [24] values obtained from a laminar flow simulation and cardiac catheterization were relatively closer to each other than the results of a turbulent flow simulation [28-30], even for stenosisaffected blood vessels [24]. This may be because most of blood flow within the entire blood flow field is considered laminar. The turbulence mode may underestimate or overestimate the simulated values. Therefore, laminar flow was set as the blood flow state in this study.

In this study, the density and dynamic viscosity of blood were $1060 \mathrm{~kg} / \mathrm{m}^{3}$ and $4 \mathrm{cP}$, respectively. On the arterial wall we imposed a no-slip boundary condition between the blood flow and the arterial wall. Furthermore, Siogkas et al. [31] reported the difference in FFR value between the rigid and elastic wall models was about $0.44 \%$ for the coronary blood flow simulation. Hence, we also assumed the arterial walls to be rigid for numerical simulations. The initial velocity and pressure were both set as $0 \mathrm{~cm} / \mathrm{s}$, and $760 \mathrm{mmHg}$ (i.e., $1 \mathrm{~atm}$ ) was taken as the reference pressure.

(a)

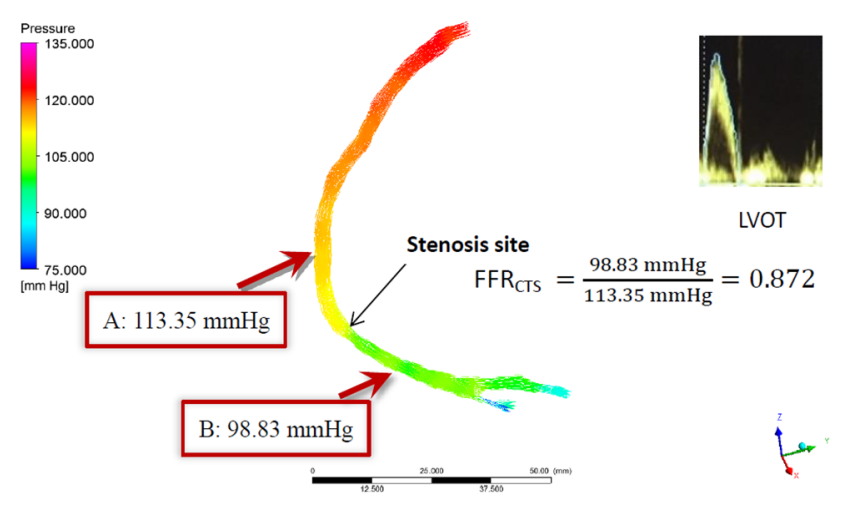

\section{Results}

\subsection{Difference between using echocardiographic data obtained for LVOT and three-lead ECG for entrance waveform setting}

In our previous research, we used the flow velocity waveform of the LVOT obtained from the patient ECG signals for the inlet flow velocity [24]. The focus of this study was the coronary blood flow condition during cardiac arrhythmia. Before further discussing the result of the blood flow condition during cardiac arrhythmia, we first compare the two simulation methods for any differences in the results for the first participant (Case I).

Figure 2 illustrates the LVOT waveform obtained from the echocardiogram and the three-lead ECG waveform for Case I. Using the patient's CCTA image from the same heartbeat, a set of 3D models for the right coronary artery (RCA) was constructed. The same cardiac rhythm, entry flow velocity, exit pressure, and vascular wall setting were modeled. In the simulation model, the periodic waveform (i.e., LVOT) at the inlet point was used as shown in Fig. 2a and the cardiac rhythm (regular cardiac rhythm) D (i.e., ECG wave form, as displayed in Fig. 2b) was simulated in the blood flow pattern.

There was a segment of RCA affected by moderate stenosis, where S2 enters S3 for Case I. The CCTA report indicated that it was 20-30\% narrower than normal arteries. By using this point of narrowing as the center, the pressure within the artery at a point $20 \mathrm{~mm}$ to the front end and $20 \mathrm{~mm}$ to the back end were measured. The ratio of these two pressures was then calculated according to the definition of FFR (i.e., the pressure at point B was divided by the pressure at point A). The ratio using the waveform obtained from LVOT as the entry flow velocity waveform was 0.872 ,

Fig. 2 FFR $_{\mathrm{CTS}}$ of in the RCA for using the $\mathbf{a}$ LVOT- and $\mathbf{b}$ ECG-based waveform

(b)

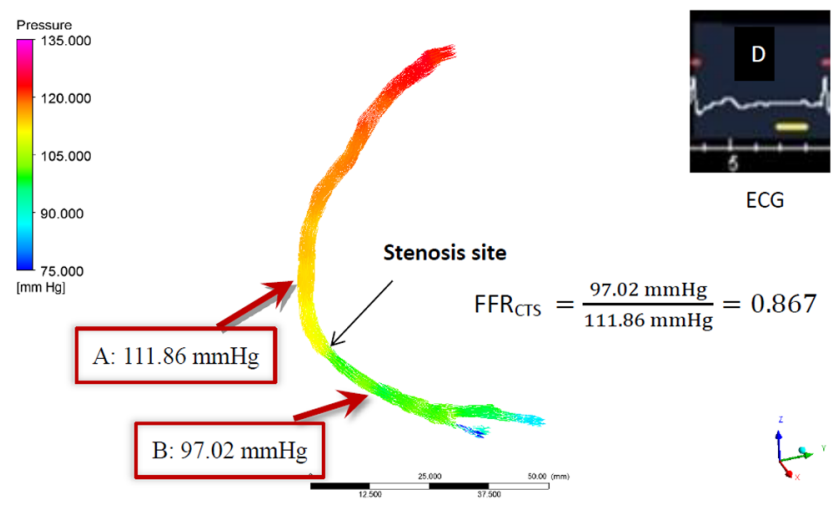


whereas that of the ECG was 0.867 . The difference was of only 0.005 , illustrating the correlation between the waveforms obtained from LVOT and ECG. Hence, the simulations thereafter used the ECG waveform obtained from CCTA scanning to model the blood flow condition during the occurrence of cardiac arrhythmia.

\subsection{Case I: blood flow and heartbeat simulation for PVC-type cardiac arrhythmia}

Case I was of PVC-type cardiac arrhythmia. The highest flow velocity for his LVOT under regular cardiac rhythm was $88 \mathrm{~cm} / \mathrm{s}$, whereas the average value was $56 \mathrm{~cm} / \mathrm{s}$. The blood pressure during cardiac CT was found to be $109 / 66 \mathrm{mmHg}$. Five heartbeats were scanned. The first four were heartbeat $A$ at $109 \mathrm{bpm}$, heartbeat $B$ at $37 \mathrm{bpm}$, heartbeat $\mathrm{C}$ at $52 \mathrm{bpm}$, and heartbeat $\mathrm{D}$ at $52 \mathrm{bpm}$. These were analyzed as in Fig. 3. The difference in patterns can be observed from the model by comparing the blood flow at the RCA. Blood was pumped out after completing the ventricular contraction process, as indicated by heartbeat $\mathrm{A}$, which should be followed by the start of the diastolic phase under normal conditions. The blood was also expected to enter the coronary arteries from the openings at the aortic root on both sides.

However, because the occurrence of PVC that led to an early contraction of the ventricles, the blood at the aortic root was pushed forward again by the blood squeezed out from the left ventricle, as indicated by heartbeat $\mathrm{B}$, before successfully entering the coronary arteries. This led to a decrease in blood flow. The blood flow measured at approximately $2.0 \mathrm{~cm}$ from the RCA entry point was $0.66 \mathrm{~mL} / \mathrm{s}$. Heartbeat B started at the point where the PVC occurred and early electrical signals were released. Because of the prior shortened diastolic phase, as indicated by heartbeat A, the blood strongly pumped out with a higher $\mathrm{R}$ wave directly from the left ventricle. Although the $\mathrm{CO}$ at this moment was relatively low, there was more time for the blood to flow into the coronary arteries, because the heartbeat that follows the early ventricular contraction will have a compensation delay to return to a normal cardiac rhythm, leading to a relatively longer diastolic period.

The blood flow measured at approximately $2.0 \mathrm{~cm}$ from the same opening of the RCA inlet was approximately $0.96 \mathrm{~mL} / \mathrm{s}$. Heartbeat $\mathrm{C}$ represented a relatively regular

(a)

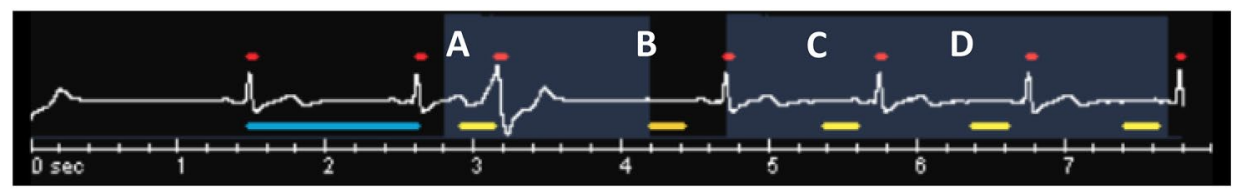

(b)

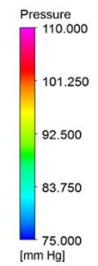

(d)

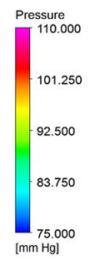

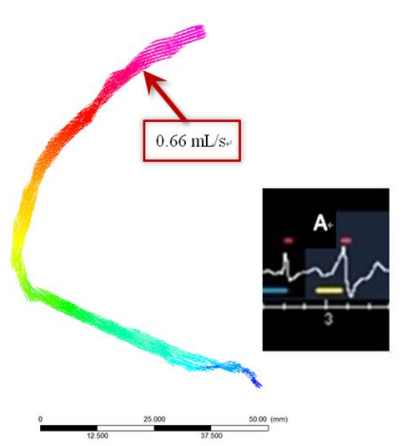

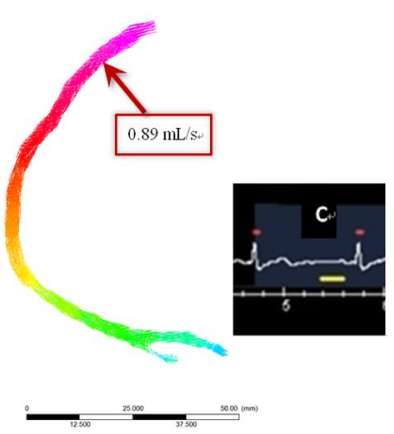

(c)

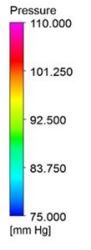

(e)

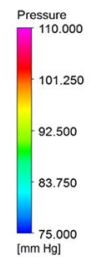

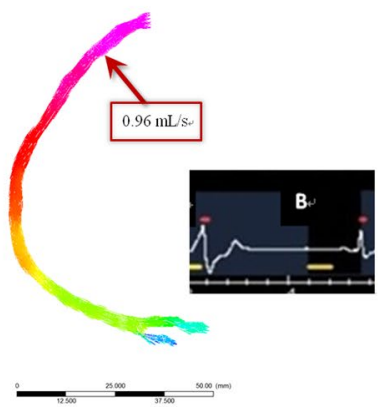

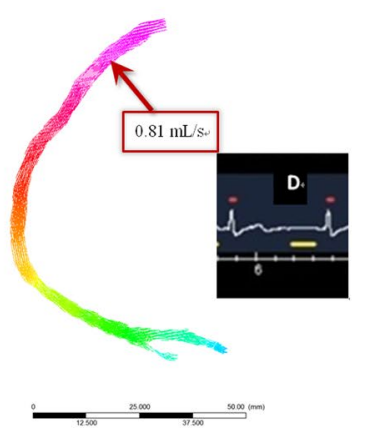

Fig. 3 Blood flow distributions in the RCA for four cardiac rhythms in Case I. a whole ECG waveform; rhythm b A, c B; d C, and e D 
cardiac rhythm from the same patient. Although this cardiac rhythm was normal, owing to the extended diastolic period from the previous heartbeats, the amount of blood replenished to the ventricles was relatively larger than normal. Hence, a larger amount of blood was also pumped out during this particular cardiac contraction. The blood flow measured at approximately $2.0 \mathrm{~cm}$ from the RCA entry point was found to be approximately $0.89 \mathrm{~mL} / \mathrm{s}$. Heartbeat $\mathrm{D}$ also represented a relatively regular cardiac rhythm from the same patient.

The diastolic period before this heartbeat was also normal. Therefore, it was expected that the amount of blood replenished to the ventricles was also at the normal value. Hence, heartbeat $\mathrm{D}$ was considered the normal heartbeat for the patient. The blood flow at approximately $2.0 \mathrm{~cm}$ from the RCA entry point was approximately $0.81 \mathrm{~mL} / \mathrm{s}$.

Table 2 Pressure gradient difference of RCA in Case II (blood pressure: $137 / 74 \mathrm{mmHg}$; LVOT max/mean: $101 / 73 \mathrm{~cm} / \mathrm{s}$ )

\begin{tabular}{lll}
\hline Heart rate $(\mathrm{bpm})$ & $\begin{array}{l}\text { Flow rate of RCA } \\
(\mathrm{mL} / \mathrm{s})\end{array}$ & $\begin{array}{l}\text { Flow rate of } \\
\text { LAD }(\mathrm{mL} / \mathrm{s})\end{array}$ \\
\hline (A) 44 & 0.72 & 1.37 \\
(B) 132 & 0.52 & 0.88 \\
(C) 46 & 0.69 & 1.35 \\
(D) 66 & 0.58 & 1.21 \\
\hline
\end{tabular}

(a)
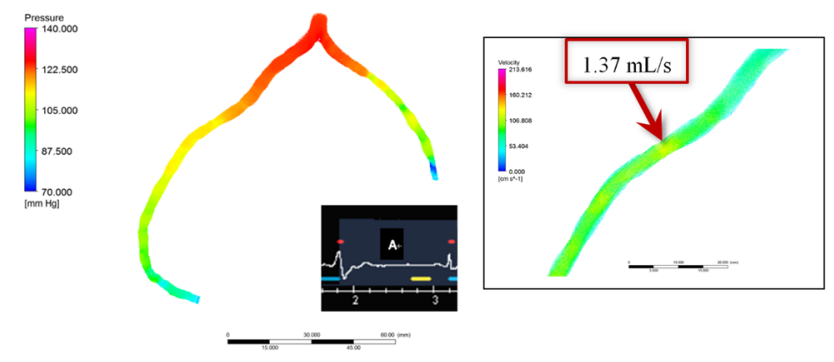

(c)

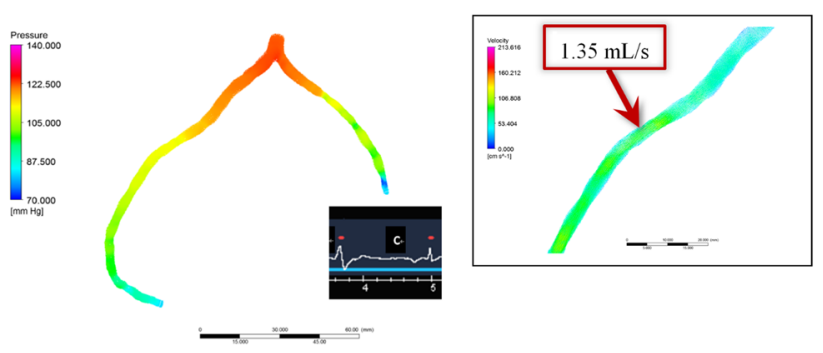

\subsection{Case II: Blood flow and heartbeat simulation for PVC-type cardiac arrhythmia}

Case II was also of PVC-type cardiac arrhythmia in addition to a history of hypertension and high cholesterol for nearly 10 years. The reason for undergoing the CCTA examination was occasional chest tightness for approximately 1 year. The highest flow rate at the LVOT under a normal heartbeat was found to be $101 \mathrm{~cm} / \mathrm{s}$, whereas the average value was $73 \mathrm{~cm} / \mathrm{s}$ (Table 2). The blood pressure was found to be $137 / 87$ during the cardiac CT. Four heartbeats were extracted from the scanning (Fig. 4). Heartbeats A, B, C, and D were found to be $44,132,46$, and $66 \mathrm{bpm}$, respectively.

In Case II, it can be observed from the CT images that there is a segment of blood vessel at the middle part of the LAD that enters the cardiac muscle, which is known as the myocardial bridge (MCB). The MCB is a common congenital anatomical deformity. From the viewpoint of the cardiac structure, the coronary arteries for normal people should be above the cardiac muscles. However, the coronary arteries for patients suffering from an MCB are within the cardiac muscles. The coronary arteries are therefore squeezed by the cardiac muscles during the systolic period and become narrower. Most patients with MCB typically show no symptoms. Some patients exhibit cardiac hypoxia during vigorous exercise. However, as the levels of hypertension, cholesterol, and blood sugar increase with age, the MCB can become increasingly detrimental.

(b)

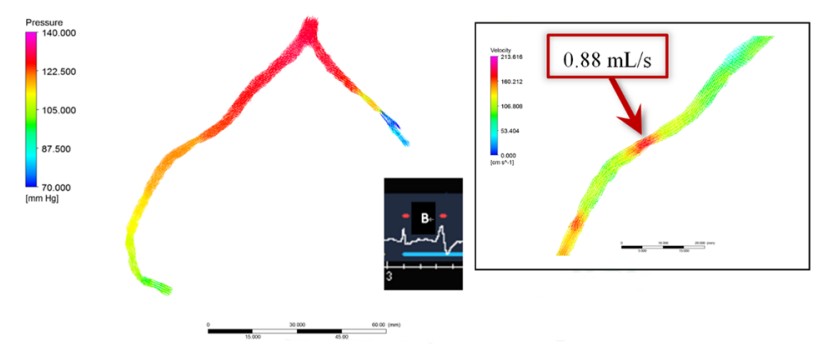

(d)
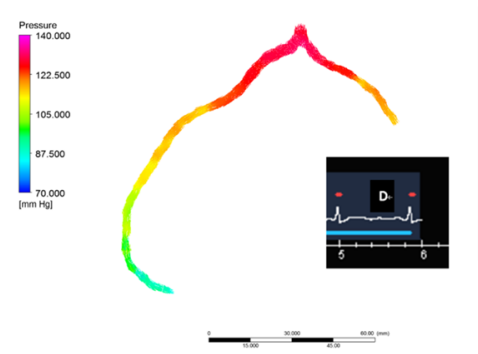

Fig. 4 Blood flow distributions in the LAD for four cardiac rhythms in Case II. Rhythms a A, b B, c C, and d D 
The simulation result indicated that the $\mathrm{LAD}$ at the $\mathrm{MCB}$ had not considerably narrowed during the first extended heartbeat A. This may be a result of the relatively long diastolic period experienced by this heartbeat, which allowed the cardiac muscle to have a longer period to relax. With a larger $\mathrm{R}$ wave rhythm, the flow rate at that point was found to be $1.37 \mathrm{~mL} / \mathrm{s}$ (Fig. 4a) (Table 2). The LAD at the MCB was found to be considerably narrowed during the second shortened heartbeat B. This may be a result of the heartbeat image being reconstructed at the end of the contraction period and the heartbeat not entering the diastolic period. The flow rate at the point of the MCB was found to be $0.88 \mathrm{~mL} / \mathrm{s}$ (Fig. 4b), as displayed in Table 2. The MCB was not found to become considerably narrower during the third heartbeat $\mathrm{C}$. The reason may be similar to that for the first heartbeat. The blood flow measured at that point was found to be $1.35 \mathrm{~mL} / \mathrm{s}$ (Fig. 4c). The fourth heartbeat $\mathrm{D}$ was found to be a normal heartbeat, where the point flow velocity at the MCB was found to be $1.21 \mathrm{~mL} / \mathrm{s}$ (Fig. 4d), as shown in Table 2. Small calcification was found only at three places in the RCA of this patient. The diameter of the blood vessels was not affected, and no notable narrowing occurred. The blood flow rates at the largest calcification during the corresponding to four heartbeats were found to be $0.72,0.52$, 0.69 , and $0.58 \mathrm{~mL} / \mathrm{s}$.

Based on the aforementioned results, a considerable blood flow decrease was noted in heartbeat $\mathrm{B}$ because of the shortened diastolic time and smaller $\mathrm{R}$ wave. Using heartbeat $\mathrm{D}$ as the reference average heartbeat, the blood flow rate of heartbeat B was only $72.73 \%$ of the flow under the average heartbeat.

\subsection{Case III: Blood flow and heartbeat simulation for AF-type cardiac arrhythmia}

Case III was of AF-type cardiac arrhythmia, with a 20-30\% stenosis at the MCB in the CT cardiac examination (Fig. 5). The largest flow velocity at the LVOT under a normal cardiac rhythm was $93 \mathrm{~cm} / \mathrm{s}$, whereas the average value was $72 \mathrm{~cm} / \mathrm{s}$. The blood pressure during cardiac CT was $130 / 84 \mathrm{mmHg}$. Four heartbeats were extracted from the scanning. Heartbeats A, B, C, and D were found to be 80 , 46, 67, and $68 \mathrm{bpm}$, respectively. The first three heartbeats were selected for further discussion focusing on the MCB of the LAD.

The blood flow measured at the MCB of the LAD for the three heartbeats in Case III was found to be $0.68,1.08$, and $1.14 \mathrm{~mL} / \mathrm{s}$. The results showed a good fit with the Case I and II simulation results, where a decrease in blood flow was also observed in the shortened cardiac rhythm. Notably, a greater decrease in blood flow was found to be possible in Case III, as the occurrence of AF could lead to a longer MCB. With heartbeat $\mathrm{C}$ as the reference average heartbeat, the blood flow for heartbeat A was only $59.65 \%$ of the normal value. Thus, AF can lead to a greater decrease in blood flow at the coronary arteries when compared with PVC. Given that patients in both Cases II and III had a narrowing point at the MCB of the LAD, (a)

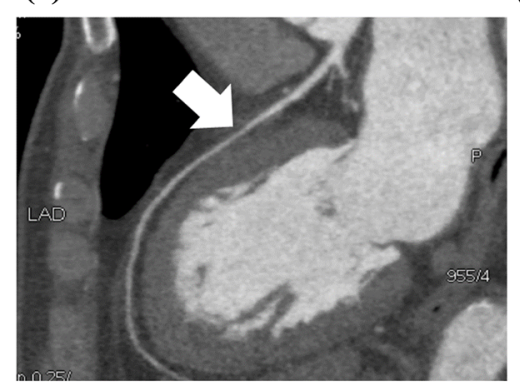

(b)

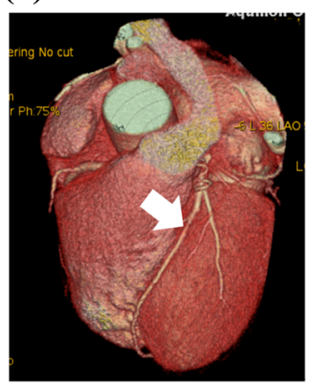

(d)

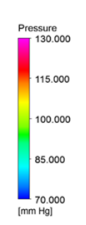

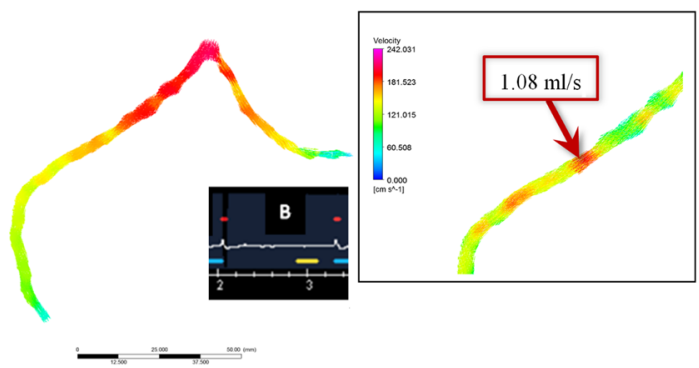

(c)

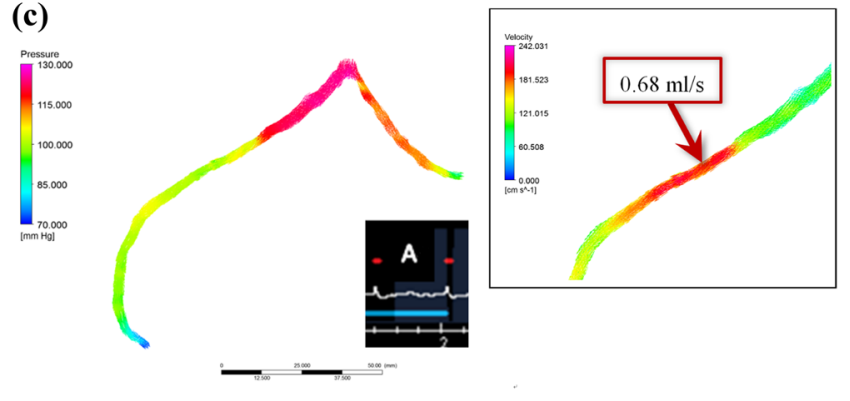

(e)
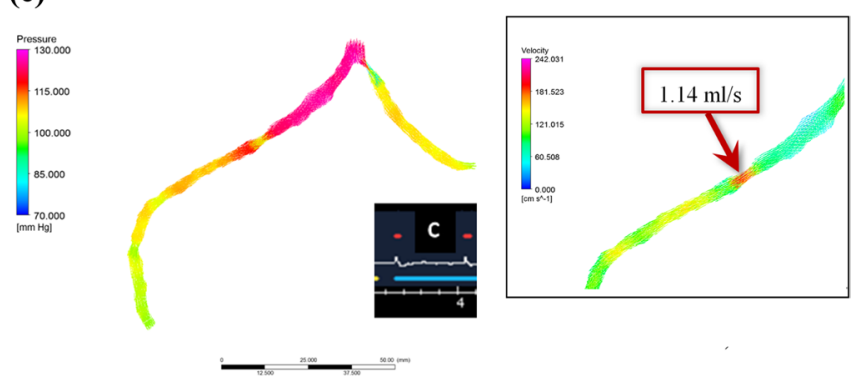

Fig. 5 Blood flow distributions in the LAD for three cardiac rhythms in Case III. a Stenosis at the LAD in CT cardiac image; $\mathbf{b}$ 3D reconstruction surface of the LAD; rhythms $\mathbf{c} A, \mathbf{d} B$, and e C 
the decrease in blood flow was different under different types of cardiac arrhythmia. The blood flow of the MCB decreased by $27.27 \%$ in Case II when PVC-type cardiac arrhythmia occurred, whereas in Case III, it decreased by $40.35 \%$ when AF-type cardiac arrhythmia occurred. This indicated that AF-type malignant cardiac arrhythmia had a stronger effect on the blood flow rate than did the PVC type.

\section{Discussion}

The ECG is noninvasive and widely used in clinical practice. As a recording of the body surface electrical activity, it provides information about heart rhythm abnormalities and helps detect CADs. However, visual inspection of the ECG provides discrete clinically interpreted features which cannot reveal the coronary blood flow information of ECG abnormalities and morphologies. Thus, this is why CFD method are required, as it can make sense of coronary blood flow and provide the ECG-based blood flow of patients during cardiac arrhythmia. Treatment for cardiac arrhythmia differs according to the type of cardiac arrhythmia and cause of the associated cardiac diseases. From the three simulation results mentioned previously, this study found that blood flow decreased regardless of whether the heartbeat was (1) an incomplete or compensatory-delayed because of early PVC or (2) shortened or lengthened because of AF where incorrect electric signals were released. In addition to studying more about the blood flow changes caused by PVC- and AF-type cardiac arrhythmia, this study aimed to extend its application to understanding other types of malignant cardiac arrhythmia, such as those related to left bundle branch block and paroxysmal supraventricular tachycardia. The preliminary results from our study demonstrated the capability of integrating electrocardiogram and image-based CFD method to demonstrate the coronary blood flow in patients during cardiac arrhythmia.

The feasibility of the integrating of the patient-specific image-based CFD method and ECG signals was tested in three arrhythmia patients. In addition, there were some limitations in the current study. First, we did not consider the motion of the coronary arteries during the cardiac cycle. In reality, the coronary arteries move significantly to contract and relax during the cardiac cycle. Second, we assumed the rigid coronary arteries for the whole computational model although we know that coronary arteries are elastic. Third, this study had a limited number of subjects recruited and should be extended by further experience. A follow-up study with a larger number of subjects may provide a better valid assessment of coronary blood flow in patients with cardiac arrhythmia.

\section{Conclusions}

In this study, we proposed a novel approach of integrating patient-specific ECG signals into CT image-based CFD simulation to analyze the blood flow in coronary arteries in patients with two types (two having premature ventricular contraction and one having atrial fibrillation) of cardiac arrhythmias. This study successfully demonstrated that cardiac arrhythmia (i.e., irregular heartbeats) can reduce the blood flow rate in the coronary arteries, especially in the presence of the AF-type cardiac arrhythmia patient.

Acknowledgements We would like to acknowledge the support from the Ministry of Science and Technology of Taiwan under the grant MOST 108-2221-E-039-010.

Author contributions All authors were actively involved in the conception, implementation, and data collection for the study. All authors met to discuss the findings of the study with respect to manuscript preparation prior to it being written. SHC was the principal author of the manuscript's first draft, produced the figures and tables, and edited subsequent revisions. CJJ clarified the idea, critically and strengthened the method of this study and edited the entire revised manuscript in terms of scientific and English writing. TCS was co-author of the manuscript's first draft. He also was the principal author involved in data analysis and helped produce many of the figures and tables. SHC, KYL, CYH, CJJ, and ZYC collected the clinical data and analyzed. TCS reviewed and edited the manuscript first draft and made significant editorial contributions to the final version which has been submitted.

\section{Compliance with ethical standards}

Conflict of interest None of the listed authors has any financial or personal relationships with other people or organizations that could potentially/inappropriately influence their work and conclusions with respect to this study or the submitted manuscript.

Open Access This article is licensed under a Creative Commons Attribution 4.0 International License, which permits use, sharing, adaptation, distribution and reproduction in any medium or format, as long as you give appropriate credit to the original author(s) and the source, provide a link to the Creative Commons licence, and indicate if changes were made. The images or other third party material in this article are included in the article's Creative Commons licence, unless indicated otherwise in a credit line to the material. If material is not included in the article's Creative Commons licence and your intended use is not permitted by statutory regulation or exceeds the permitted use, you will need to obtain permission directly from the copyright holder. To view a copy of this licence, visit http://creativecommons.org/licenses/ by $/ 4.0 \%$.

\section{References}

1. Benjamin, E. J., Muntner, P., Alonso, A., Bittencourt, M. S., Callaway, C. W., Carson, A. P., et al. (2019). Heart disease and stroke statistics-2019 update: a report from the American Heart Association. Circulation, 139(10), e52-e528. https://doi.org/10.1161/ CIR.0000000000000659. 
2. Joseph, P., Leong, D., McKee, M., Anand, S. S., Schwalm, J. D., Teo, K., et al. (2017). Reducing the global burden of cardiovascular disease, part 1: The epidemiology and risk factors. Circulation Research, 121(6), 677-694.

3. Sones, M. F., Jr., \& Shirey, E. K. (1962). Cine coronary arteriography. Modern Concepts of Cardiovascular Disease, 31, 735-738.

4. Pijls, N. H., van Schaardenburgh, P., Manoharan, G., Boersma, E., Bech, J. W., van't Veer, M., et al. (2007). Percutaneous coronary intervention of functionally nonsignificant stenosis: 5-year follow-up of the DEFER Study. Journal of the American College of Cardiology, 49(21), 2105-2111.

5. Pijls, N. H. J., van Son, J. A., Kirkeeide, R. L., de Bruyne, B., \& Gould, K. L. (1993). Experimental basis of determining maximum coronary, myocardial, and collateral blood flow by pressure measurements for assessing functional stenosis severity before and after percutaneous transluminal coronary angioplasty. Circulation, 86(4), 1354-1367.

6. Pijls, N. H. J., \& Sels, J. W. (2012). Functional measurement of coronary stenosis. Journal of the American College of Cardiology, 59(12), 1045-1057. https://doi.org/10.1016/j.jacc.2011.09.077.

7. Tonino, P. A., de Bruyne, B., Pijls, N. H. J., et al. (2009). Fractional flow reserve versus angiography for guiding percutaneous coronary intervention. The New England Journal of Medicine, 360(3), 213-224.

8. Zhang, J. M., Zhong, L., Luo, T., Lomarda, A. M., Huo, Y., et al. (2016). Simplified models of non-invasive fractional flow reserve based on CT images. PLOS ONE, 11(5), e0153070. https://doi. org/10.1371/journal.pone.0153070.

9. Tan, X. W., Zheng, Q., Shi, L., Gao, F., et al. (2017). Combined diagnostic performance of coronary computed tomography angiography and computed tomography derived fractional flow reserve for the evaluation of myocardial ischemia: A meta-analysis. International Journal of Cardiology, 236(2017), 100-106.

10. Arvanaghi, R., Daneshvar, S., Seyedarabi, H., \& Goshvarpour, A. (2017). Fusion of ECG and ABP signals based on wavelet transform for cardiac arrhythmias classification. Computer Methods and Programs in Biomedicine, 151, 71-78.

11. Elhaj, F. A., Salim, N., Harris, A. R., Swee, T. T., \& Ahmed, T. (2016). Arrhythmia recognition and classification using combined linear and nonlinear features of ECG signals. Computer Methods and Programs in Biomedicine, 127, 52-63.

12. Raj, S., Ray, K. C., \& Shankar, O. (2016). Cardiac arrhythmia beat classification using DOST and PSO tuned SVM. Computer Methods and Programs in Biomedicine, 136, 163-177.

13. Lyon, A., Mincholé, A., Martínez, J. P., Laguna, P., \& Rodriguez, B. (2018). Computational techniques for ECG analysis and interpretation in light of their contribution to medical advances. Journal of the Royal Society, Interface, 15(138), 20170821. https ://doi.org/10.1098/rsif.2017.0821.

14. Berkaya, S. K., Uysal, A. K., Gunal, E. S., Ergin, S., Gunal, S., \& Gulmezoglu, M. B. (2018). A survey on ECG analysis. Biomedical Signal Processing and Control, 43, 216-235.

15. Pławiak, P. (2018). Novel methodology of cardiac health recognition based on ECG signals and evolutionary-neural system. Expert System with Applications, 92, 334-348.

16. Taylor, C. A., \& Draney, M. T. (2004). Experimental and computational methods in cardiovascular fluid mechanics. Annual Review of Fluid Mechanics, 36, 197-231.

17. Taylor, C. A., Fonte, T. A., \& Min, J. K. (2013). Computational fluid dynamics applied to cardiac computed tomography for noninvasive quantification of fractional flow reserve: Scientific basis. Journal of the American College of Cardiology, 61, 2233-2241.
18. Kim, H. J., Vignon-Clementel, I. E., Coogan, J. S., Figueroa, C. A., Jansen, K. E., \& Taylor, C. A. (2010). Patient-specific modeling of blood flow and pressure in human coronary arteries. Annals of Biomedical Engineering, 38(10), 3195-3209.

19. Miyoshi, T., Osawa, K., Ito, H., Kanazawa, S., Kimura, T., Shiomi, H., et al. (2015). Non-invasive computed fractional flow reserve from computed tomography $(\mathrm{CT})$ for diagnosing coronary artery disease-Japanese results from NXT trial (Analysis of Coronary Blood Flow Using CT Angiography: Next Steps). Circulation Journal, 79(2), 406-412.

20. Koo, B. K., Erglis, A., Doh, J. H., Daniels, D. V., Jegere, S., Kim, H. S., et al. (2011). Diagnosis of ischemia-causing coronary stenoses by noninvasive fractional flow reserve computed from coronary computed tomographic angiograms. Results from the prospective multicenter DISCOVER-FLOW (Diagnosis of Ischemia-Causing Stenoses Obtained Via Noninvasive Fractional Flow Reserve) study. Journal of the American College of Cardiology, 58(19), 1989-1997.

21. Min, J. K., Leipsic, J., Pencina, M. J., Berman, D. S., Koo, B. K., et al. (2012). Diagnostic accuracy of fractional flow reserve from anatomic CT angiography. JAMA, 308(12), 1237-1245.

22. Min, J. K., Taylor, C. A., Achenbach, S., Koo, B. K., Leipsic, J., Nørgaard, B. L., et al. (2015). Noninvasive fractional flow reserve derived from coronary $\mathrm{CT}$ angiography: Clinical data and scientific principles. JACC: Cardiovascular Imaging, 8(10), $1209-1222$.

23. Tu, S., Barbato, E., Köszegi, Z., Yang, J., Sun, Z., Holm, N. R., et al. (2014). Fractional flow reserve calculation from 3-dimensional quantitative coronary angiography and TIMI frame count: A fast computer model to quantify the functional significance of moderately obstructed coronary arteries. JACC: Cardiovascular Interventions, 7(7), 768-777.

24. Lin, K. Y., Shih, T. C., Chou, S. H., Chen, Z. Y., Hsu, C. H., \& Ho, C. Y. (2016). Computational fluid dynamics with application of different theoretical flow models for the evaluation of coronary artery stenosis on CT angiography: Comparison with invasive fractional flow reserve. Biomedical Physics and Engineering Express, 2(6), 065011.

25. Kwon, S. S., Chung, E. C., Park, J. S., Kim, G. T., Kim, J. W., Kim, K. H., et al. (2014). A novel patient-specific model to compute coronary fractional flow reserve. Progress in Biophysics and Molecular Biology, 116(1), 48-55.

26. Sakamoto, S., Takahashi, S., Coskun, A. U., Papafaklis, M. I., Takahashi, A., Saito, S., et al. (2013). Relation of distribution of coronary blood flow volume to coronary artery dominance. The American Journal of Cardiology, 111(10), 1420-1424.

27. White, F. M. (2011). Fluid mechanics (7th ed.). New York: McGraw-Hill.

28. Launder, B. E., \& Spalding, D. B. (1974). The numerical computation of turbulent flows. Computer Methods in Applied Mechanics and Engineering, 3(2), 269-289.

29. Menter, F. R. (1994). Two-equation eddy-viscosity turbulence models for engineering applications. American Institute of Aeronautics and Astronautics Journal, 32(8), 1598-1605.

30. Wilcox, D. C. (1988). Reassessment of the scale-determining equation for advanced turbulence models. American Institute of Aeronautics and Astronautics Journal, 26(11), 1299-1310.

31. Siogkas, P. K., Papafaklis, M. I., Sakellarios, A. I., Stefanou, K. A., Bourantas, C. V., Athanasiou, L. S., et al. (2015). Patientspecific simulation of coronary artery pressure measurements: An in vivo three-dimensional validation study in humans. BioMed Research International, 2015, , 628416. 